\title{
Re: Appendiceal Substitution Following Right Proximal Ureter Injury
}

To the Editor,

Use of the appendix as a ureteral substitute was first described by Melnikoff in 1912 (1). However, the technique has been used only in a handful of patients since its introduction $(2,3)$. We present the case of a 66 year-old male who presented with abdominal pain three weeks after undergoing lysis of small bowel adhesions, and was found to have an $8-10 \mathrm{~cm}$ defect of the right proximal ureter upon undergoing retrograde pyelogram.

There are numerous techniques for the repair of ureteral injuries. Primary end-to-end anastomosis, psoas hitch ureteral reimplantation, and Boari flap were not feasible in this case due to the length and location of the injury. Ileal interposition has been successfully used to repair large defects, but requires a bowel anastomosis, which we wished to avoid. Auto-transplantation of the kidney is technically challenging and associated with unique morbidities. Appendiceal substitution was chosen due to the amenable location of the injury and favorable operative risks.

In the operating room, we injected methylene blue through a previously placed nephrostomy tube in order to better delineate the proximal margin of the injury. The appendix was then ligated at its base and

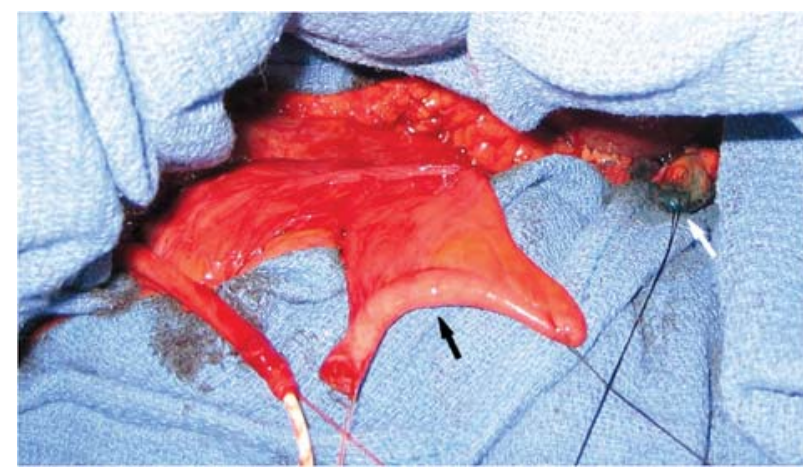

Figure 1 - Proximal ureteral defect measuring $8-10 \mathrm{~cm}$ with appendix rotated into position (black arrow). Dark suture at right renal pelvis (white arrow). tip and detached from the cecum. Special attention was given to preserving the appendicular arteries and mesoappendix (Figure-1). The appendix was cannulated to accommodate a 14 French endopyelotomy stent. Next, the appendix was rotated up to the level of the renal pelvis to ensure a tension free anastomosis. It was then oriented in isoperistaltic fashion with its distal tip abutting the renal pelvis. A spatulated ureteroappendiceal anastomosis was performed on both ends of the graft (Figure-2). The anastomosis was then tested

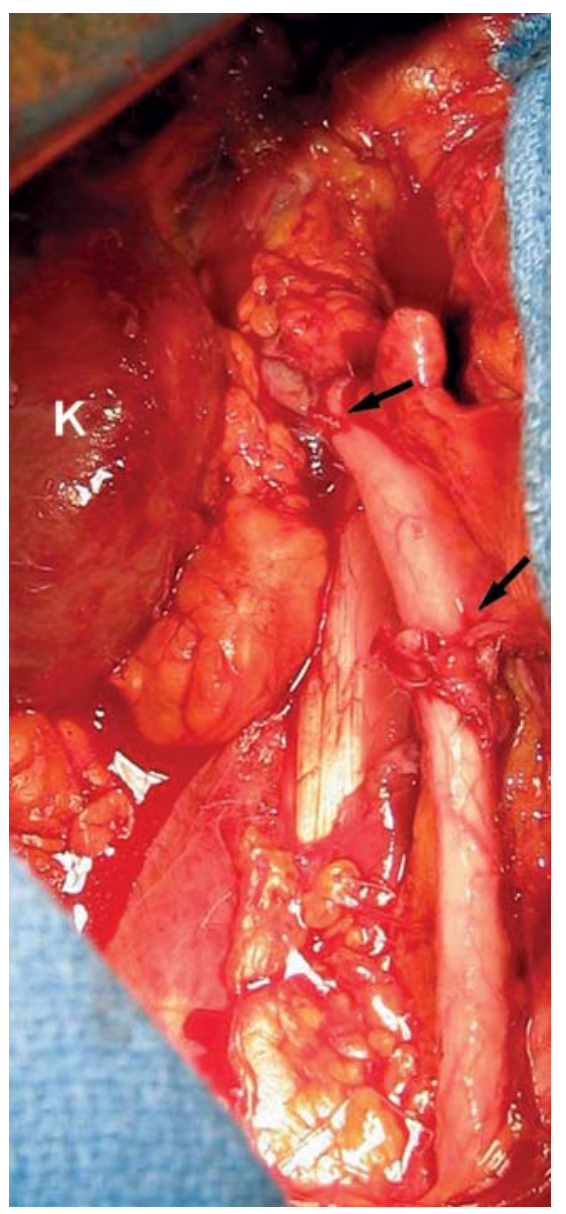

Figure 2 - Completed appendiceal interposition (arrows). $K=$ right kidney. 


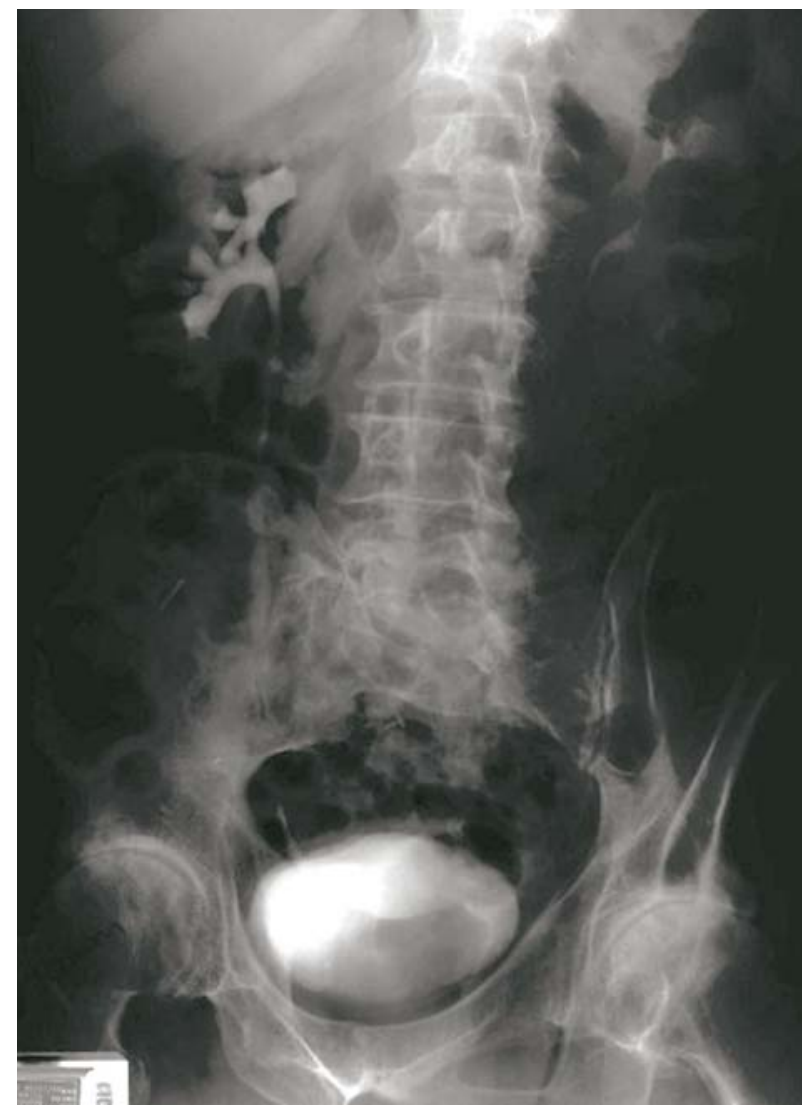

Figure 3 - Intravenous pyelogram at eight months postoperatively showing a patent graft with no evidence of stricture or hydronephrosis.

for leakage by injecting methylene blue through the indwelling nephrostomy tube.

The patient was discharged from the hospital on postoperative day six and the stents were removed four weeks later. Intravenous pyelogram at eight months postoperatively showed a patent appendiceal graft with no evidence of stricture or hydronephrosis (Figure-3).
Long-term data in the small body of literature devoted to this procedure demonstrates excellent autograft performance and preserved renal function up to fifteen years postoperatively (2). While traumatic injury is the most commonly reported indication for this procedure, it has also been employed successfully in other settings such as ureteral necrosis secondary to dermatomyositis. This technique has also been proven effective in pediatric as well as adult populations (2).

The majority of case reports of appendiceal interposition involve the right ureter due to the ipsilateral location of the appendix. However, there is at least one description of a proximal left ureteral repair by Zargar et al. 2004 (3). To accomplish the left-sided reconstruction the author mobilized the appendix with the right colon and distal ileum into the left ureteral fossa.

This case supports appendiceal substitution as a reasonable option for patients with right-sided ureteral defects not amenable to primary end-toend anastomosis. Limiting factors for the procedure include presence and length of appendix, impaired renal function, and history of pelvic irradiation.

\section{REFERENCES}

1. Melnikoff AE: Sur le replacement de l'uretere par anse isolée de l'intestin grêle. Rev Clin Urol. 1912; 1: 60105.

2. Richter F, Stock JA, Hanna MK: The appendix as right ureteral substitute in children. J Urol. 2000; 163: 190812.

3. Zargar MA, Mirzazadeh M, Zargar K: The appendix, an acceptable substitute for all segments of both ureters: a report of two cases. Med J Islam Repub Iran. 2004; 18: 177-180.

Matt S. Ashley, BA \& Dr. Siamak Daneshmand Division of Urology \& Renal Transplantation Oregon Health \& Science University Portland, Oregon, USA E-mail:daneshma@ohsu.edu 